\title{
Reorganization of Exuberant Axonal Arbors Contributes to the Development of Laminar Specificity in Ferret Visual Cortex
}

\author{
Victor Borrell and Edward M. Callaway \\ Systems Neurobiology Laboratories, The Salk Institute for Biological Studies, 10010 North Torrey Pines Road, La Jolla, \\ California 92037
}

\begin{abstract}
Layer-specific cortical axons are believed to develop precisely from the outset without making exuberant branches in incorrect cortical layers. We tested this hypothesis by following the development of axonal arbors of layer $2 / 3$ pyramidal neurons in ferret visual cortex, which in the adult have dense axonal arbors in layers $2 / 3$ and 5 and not in layer 4 . We began our studies at earlier ages and sampled at closer intervals than in previous studies. Our studies reveal that between postnatal day (P) 14 and P18 the initial growth of axonal arbors lacks specificity for layers $2 / 3$ and 5 and involves the formation of a small number of branches incorrectly in layer 4 . After P18 the number of incorrect axonal branches in layer 4 decreased, whereas there was extensive axonal growth specifically in layers $2 / 3$ and 5 . To further study the mechanisms involved in directing the layer-
\end{abstract}

specific growth and elimination of axonal branches, we studied the development of layer 2/3 neurons in slices kept in culture for $5 \mathrm{~d}$. In these studies the initial imprecise growth at P14-18 followed by precise growth after P18 was mimicked in vitro, but the elimination of incorrect axons from layer 4 did not occur. These studies reveal that most axonal arbors grow precisely in the correct layers, but the initial growth involves the formation of a small number of incorrect axonal branches that are later eliminated. Therefore the mechanisms that regulate axonal growth are not as precise initially as later, and mechanisms must exist to allow selective elimination of axon collaterals from incorrect layers.

Key words: local circuits; axonal branching; layer 2/3; pyramidal cells; visual cortex; ferret
The correct function of the nervous system requires the formation of complex neural networks, which interconnect thousands of cells of many different types in exquisitely precise patterns. A common feature in the development of neural circuits is the formation of axonal arbors in precise target areas, with the exclusion of other nearby non-target areas (Goodman and Shatz, 1993). Numerous studies have shown that in the adult cerebral cortex, neurons in different layers have elaborate axonal arbors within restricted target layers, excluding non-target layers. For example, pyramidal cells in layers $2 / 3$ and 5 arborize their axons preferentially within layers $2 / 3$ and 5 , excluding layers 4 and 6 (Lund and Boothe, 1975; Callaway and Wiser, 1996; Callaway, 1998a) (for review, see Gilbert, 1983; Callaway, 1998b). It has been shown that in ferret, cat, monkey, and human, layer-specific local axonal arbors develop precisely, apparently without making incorrect branches that might later be pruned (Lund et al., 1977; Katz, 1991; Burkhalter et al., 1993; Callaway and Lieber, 1996; Callaway, 1998a) (for review, see Katz and Callaway, 1992; Callaway, 1998b). The results of these studies have important implications for the types of mechanisms involved. For example, these findings suggest that cortical axons respond to layer-specific molecular cues intrinsic to the cortical layers to elaborate their local circuits and also imply that these cues are already present at the

Received Dec. 25, 2001; revised May 2, 2002; accepted May 15, 2002.

This work was supported by National Institutes of Health Grant EY10742. The Human Frontier Science Program Organization provided postdoctoral support for V.B. We thank Dr. H. A. E. Lechner, Dr. A. K. Butler, and T. L. V. Martinez for technical assistance, and D. D. Larsen for helpful comments on this manuscript.

Correspondence should be addressed to Victor Borrell, The Salk Institute for Biological Studies, SNL-C, 10010 North Torrey Pines Road, La Jolla, CA 92037. E-mail: borrell@salk.edu.

Copyright (C) 2002 Society for Neuroscience $\quad 0270-6474 / 02 / 226682-\bullet \$ 15.00 / 0$ time of initial axon outgrowth (Katz and Callaway, 1992; Goodman and Shatz, 1993).

In further support of the hypothesis that layer-specific molecular cues instruct growing axons to arborize in the correct layers, normal laminar specificity can develop in cultured slices of ferret visual cortex. In particular, layer 6 pyramidal neurons grow axons preferentially in layers 4 and 6 (Dantzker and Callaway, 1998), whereas layer 5 pyramids grow axons predominantly in layers $2 / 3$ and 5, avoiding layer 4 (Butler et al., 2001).

Surprisingly, however, many layer $2 / 3$ pyramidal neurons fail to grow layer-specific axons in the same slice cultures (Butler et al., 2001). These results led to the hypothesis that slice cultures do not preserve the conditions necessary for specific growth of layer $2 / 3$ pyramids (Butler et al., 2001). Another possibility is that the lack of layer-specific growth of layer $2 / 3$ neurons resulted from immaturity of the cortex when cultures were made. Consistent with this possibility, we report here that layer $2 / 3$ pyramidal neurons developing in slice cultures prepared from more mature animals were able to grow layer-specific axons in vitro.

These observations further suggested that layer $2 / 3$ pyramidal neurons developing in vivo might also fail to form axonal arborizations as precisely at the earliest stages of growth as they do later. We therefore analyzed layer $2 / 3$ pyramids that had developed in ferret visual cortex in vivo and sampled them at earlier and closer time points than were used in previous studies. These cells were labeled by transfection and expression of green fluorescent protein (GFP) for $24 \mathrm{hr}$ in vitro. We find that there is a brief time window when small numbers of axonal branches are formed incorrectly in layer 4 and then eliminated. Nevertheless, the great majority of axonal branches are formed later, and at these ages they precisely target the correct layers, $2 / 3$ and 5 . The period of most exuberant axonal growth is also correlated with 
extensive dendritic growth, suggesting a link between these processes.

\section{MATERIALS AND METHODS}

Visual cortex slice cultures. Ferrets were obtained from Marshall Farms (North Rose, NY) and kept on a $12 \mathrm{hr}$ light/dark cycle. All animals were treated in accordance with institutional and NIH guidelines for the Care and Use of Laboratory Animals.

Postnatal day (P) $14(n=4), \mathrm{P} 18(n=4), \mathrm{P} 22(n=5), \mathrm{P} 26(n=3)$, and P30 $(n=3)$ ferrets from six different litters were deeply anesthetized with sodium pentobarbital (100 mg/kg i.p.) and decapitated. Organotypic slices of visual cortex were prepared as described previously, under sterile conditions (McAllister et al., 1995; Callaway and Lieber, 1996; Butler et al., 2001). Briefly, the brain was removed and submerged in ice-cold HEPES-buffered artificial CSF (ACSF) containing (in mM): 140 $\mathrm{NaCl}, 5 \mathrm{KCl}, 1 \mathrm{MgCl}_{2}$, 24 D--glucose, 10 HEPES, $1 \mathrm{CaCl}_{2}, \mathrm{pH}$ 7.2. The pia was removed using fine forceps, and the tissue from the posterior pole of each hemisphere was cut into a single block containing area 17 of the visual cortex. This block was then sliced sagittally to a thickness of 400 $\mu \mathrm{m}$ using a tissue slicer (Katz, 1987). Slices were then placed onto cell culture inserts $(0.4 \mu \mathrm{m}$ pore size, Fisher) overlying $1 \mathrm{ml}$ of medium. Culture medium was composed of 50\% Eagle's basal medium, $25 \%$ HBSS, $25 \%$ horse serum, $330 \mathrm{~mm}$ D-glucose, $10 \mathrm{~mm}$ HEPES, and $10 \mathrm{U} / \mathrm{ml}$ penicillin-streptomycin (all from Invitrogen). Slices were then incubated and maintained in a $95 \%$ humidity, $5 \% \mathrm{CO}_{2}$ atmosphere at $37^{\circ} \mathrm{C}$.

Biolistic transfection and tissue processing. To reveal the morphology of pyramidal cells, within $1 \mathrm{hr}$ after preparation the slices were transfected under sterile conditions using a Biolistics Helios gene gun device (Bio$\mathrm{Rad}$ ). This method of transfection has been shown previously not to alter dendritic morphology or the layer specificity of axonal projections in transfected pyramidal cells in ferret visual cortex (McAllister et al., 1995; Butler et al., 2001). Gene gun cartridges were prepared by precipitating $50 \mu \mathrm{g}$ of a CMVP-eGFP-N1 plasmid (Clontech, Palo Alto, CA) onto $12.5 \mathrm{mg}$ of $1.6 \mu \mathrm{m}$ gold particles, which were then used to coat $1 \mathrm{~m}$ of tubing (Bio-Rad). By application of a helium pressure of 80 psi, DNAcoated gold particles were accelerated into the tissue. Slices were immediately returned to the incubator.

After $24 \mathrm{hr}$ of culture, or $5 \mathrm{~d}$ in vitro (DIV), slices were fixed with $2.5 \%$ paraformaldehyde, $4 \%$ sucrose in $0.1 \mathrm{M}$ phosphate buffer (PB), $\mathrm{pH} 7.4$, for $1.5 \mathrm{hr}$ at room temperature. Then they were cryoprotected in $30 \%$ sucrose in $0.1 \mathrm{M} \mathrm{PB}$, freeze-thawed, and incubated with blocking solution (10\% normal goat serum, $2 \%$ bovine serum albumin, $0.25 \%$ Triton $\mathrm{X}-100$, in $0.1 \mathrm{M} \mathrm{PB})$. Visualization of eGFP was enhanced by using a polyclonal anti-GFP antibody (Molecular Probes, Eugene, OR) diluted at 1:500 in blocking solution, followed by a Cy3-conjugated secondary antibody (1:100; Chemicon, Temecula, CA). In addition, slices were counterstained with 4',6-diamidino-2-phenylindole (DAPI) $(20 \mu \mathrm{M}$; Sigma) to allow determination of laminar borders. Finally, slices were mounted onto subbed slides, dehydrated, and coverslipped in Krystalon (Fisher).

Cells labeled with GFP in slice cultures for $24 \mathrm{hr}$ were used as a measure of the developmental state in vivo at the time that cultures were prepared. This was done because the use of GFP labeling by cell transfection to reveal neuronal processes has the limitation that a minimum incubation time of $24 \mathrm{hr}$ is needed to allow expression and intracellular diff usion of the GFP. Nonetheless, it is reasonable to assume that the morphology of axonal and dendritic arbors after $24 \mathrm{hr}$ in vitro is very similar to that in vivo on the day that slice cultures are made. As detailed further in Discussion, our findings cannot be attributed to changes that occurred during the $24 \mathrm{hr}$ culture period.

Cell selection and reconstruction. Tissue slices were analyzed only if visual cortical area 17, and layers within it, could be identified unequivocally. In addition, to avoid drawing cells in slices cut obliquely, where axons and dendrites may have been cut off close to the cell body, slices were selected only if a majority of pyramidal cells appeared healthy, and within those slices, only cells with a descending axon that could be followed down to the white matter were drawn.

Cells were reconstructed by using a $40 \times$ oil immersion objective [1.3 numerical aperture (NA)] on a Nikon fluorescence microscope in concert with a Neurolucida computerized reconstruction system (MicroBrightField, Colchester, VT). First, low-power $(10 \times$ objective, 0.5 NA) maps were drawn of each selected slice. Maps included locations of the cell bodies of all labeled cells, area 17 borders, and cortical layer borders. Then, axonal and dendritic arbors of selected cells were reconstructed at higher power without the knowledge of cortical layers or area 17 borders (i.e., "drawn blind"). After completion, neuronal reconstructions were placed into the lower-power map file for analysis.

Pyramidal cells were drawn only if they met the following criteria: (1) they were within area 17; (2) they were brightly labeled and not too close to another labeled cell, to allow accurate reconstruction of axon and dendrites; (3) they appeared healthy (as determined by the absence of notable membrane blebbing in the cell body, axons, or dendrites); and (4) they had a pyramidal morphology and descending axon originating from the base of the cell body that extended through all cortical layers and into the white matter. Although we selected pyramidal cells with the main descending axon uncut, ensuring that all primary axonal branches are preserved, it is likely that during the process of slice making some secondary collaterals were lost. However, this cannot account for the decrease in layer 4 axonal branches detected after P18 (see Results), because most axonal branches detected in layer 4 at this stage were primary branches (37 of 40 branches; $93 \%$ ).

Quantification and data analysis. The number of axonal branch points within individual layers of area 17 was analyzed by using a customdesigned Matlab-based program. Axonal branches were defined as primary if they originated from the main efferent axon; otherwise they were considered as nonprimary. To analyze the spatial distribution of axonal branches within layers 4 and 5, the thickness of these layers was subdivided into five equal portions (named A through E), each representing $20 \%$ of the depth; then the number of axonal branch points within each of the portions was counted. The number of basal dendrites, of apical and basal dendritic branch points, as well as total dendritic length, was calculated with the Neurolucida Winmorph analysis program (MicroBrightfield). All data were statistically analyzed by using one-way ANOVA (for multiple group comparison) and Student-Newman-Keuls test or $t$ test (for pair-wise comparisons); $p<0.05$ was considered significant.

Calculation of dendrite growth index. To summarize the changes in dendritic morphology between developmental stages, we used the data on dendritic form shown in Table 1 to calculate the dendrite growth index (DGI). We used a modification of the Dendrite Modification Index described by McAllister et al. (1995). The DGI was calculated as:

$$
\mathrm{DGI}=\sum_{i=1}^{3} C_{\mathrm{i}} F_{\mathrm{i}} / I_{\mathrm{i}},
$$

where $F_{\mathrm{i}}$ is the value of a given parameter "i" at the final of the stages compared, $I_{\mathrm{i}}$ is the value at the initial stage, and $C_{\mathrm{i}}$ is a normalized weighting constant the magnitude of which depends on the parameter. For basal dendrites, we assigned $C_{1}=0.4$ for total length of dendrite, $C_{2}$ $=0.3$ for number of basal dendrites, and $C_{3}=0.3$ for number of dendritic branches. The ratio $F / I$ was set to a value of 1 for cases in which final values were not statistically distinct from initial values $(p<0.05)$. The DGI for apical dendrites was calculated similarly, although because the number of apical dendrites is always 1 , there were only two parameters: $C_{1}=0.5$ for total length of dendrite and $C_{2}=0.5$ for number of dendritic branches. According to these formulas, a DGI smaller than 1 means a significant decrease in dendritic complexity, and a DGI larger than 1 means a significant increase in complexity. Also, a larger value of DGI represents a greater increase in dendritic complexity.

\section{RESULTS}

We examined the development of local layer-specific axonal projections of layer $2 / 3$ pyramidal neurons in area 17 of ferret visual cortex. Cortical slices were prepared from P14 to P30 ferrets. Pyramidal neurons were intracellularly labeled in slices by using Biolistic transfection with a GFP-encoding plasmid. In one set of experiments, neurons that had developed in vivo were labeled with GFP during $24 \mathrm{hr}$ in vitro. In another set of experiments, GFP-labeled neurons developed in slice cultures in vitro for $5 \mathrm{~d}$ before analysis. P14 was the earliest age examined because it is the stage at which neurons committed to form layer $2 / 3$ begin to accumulate above the presumptive layer 4 (Jackson et al., 1989), and at this age axonal arbors are very immature (Callaway and Lieber, 1996; Dantzker and Callaway, 1998; Butler et al., 2001).

The GFP transfection method clearly labeled dendrites and axons after a period of $\sim 24 \mathrm{hr}$ (Fig. 1A). Axons could be followed for long distances (up to millimeters) and throughout the depth of 

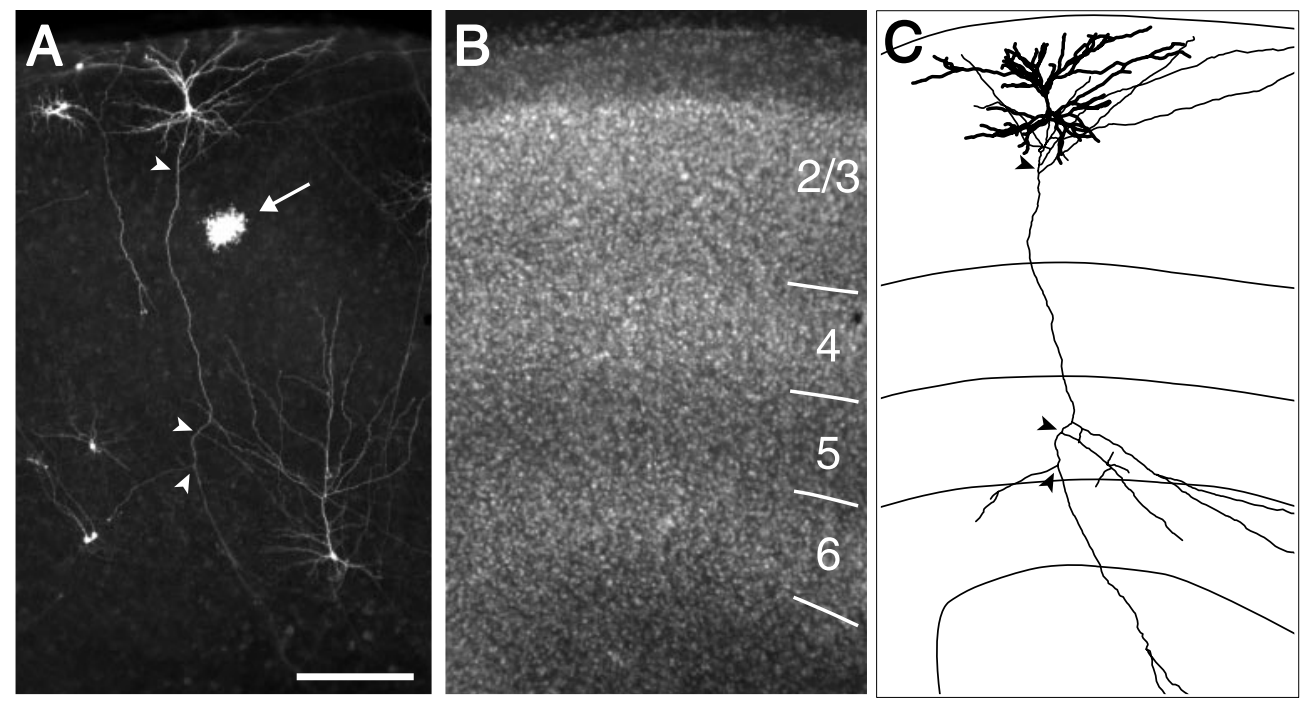

Figure 1. Intracellular labeling and neuronal reconstruction. $A, B$, Low-power fluorescence photomicrographs of an area 17 slice culture from a P30 ferret showing several GFP-labeled cells $24 \mathrm{hr}$ after transfection $(A)$ and the DAPI counterstaining of the same area $(B)$, where cortical layers can be identified (numbers to right). Two pyramidal cells, in layers $2 / 3$ and 6 , are visible in $A$ (top center and bottom right, respectively). The layer $2 / 3$ pyramid displays a descending axon that branches several times in layers $2 / 3$ and 5 (white arrowheads) but not in layer 4 . Occasionally, glial cells were also labeled (arrow). $C$, Camera lucida reconstruction of the layer $2 / 3$ pyramidal neuron shown in $A$, overlapped with the laminar borders identified with the DAPI staining shown in $B$. Thick lines represent the dendritic arbor, and thin lines represent the axonal arbor. Black arrowheads identify the same axonal branch points indicated in $A$. Scale bar (shown in $A$ for $A-C$ ): $200 \mu \mathrm{m}$.

the slice and were often capped with growth cone-like structures (data not shown), indicating that this labeling method is effective to visualize the entire axonal arbor present within the slice [see also Butler et al. (2001)]. Cortical layers were determined by counterstaining with DAPI (Fig. $1 B$ ). The number of labeled pyramidal neurons per slice varied greatly, from 1 to $>50$, but always a majority $(>90 \%)$ of the transfected cells appeared healthy and displayed extensive and brightly stained axonal and dendritic arbors, which could then be reconstructed (see Materials and Methods) (Fig. 1A,C). Occasionally, interneurons (data not shown) and glial cells (Fig. $1 A$ ) were also transfected, but these were not included in our analyses.

Our interest in the in vivo development of laminar specificity of axonal arbors of layer $2 / 3$ pyramidal neurons was originally motivated by our findings from neurons that developed in vitro for $5 \mathrm{~d}$. With these we find that development of laminar specificity in vitro depends on the age when slice cultures are made. Layer $2 / 3$ pyramidal neurons develop layer-specific axonal arbors in vitro only in slices obtained from P18 or older ferrets, but not from P14 ferrets (see details below). This suggests that axonal arbors growing in vivo between P14 and P18 may also lack laminar specificity. Nevertheless, the details of the findings for neurons developing in vitro (5 DIV) can be described and interpreted more clearly following description of the developmental events that occur in vivo. We therefore begin with a detailed description of neurons that developed in vivo before labeling.

\section{Development of axonal arbors in vivo}

In the following analysis we have assumed that the anatomical characteristics of our samples labeled with GFP for $24 \mathrm{hr}$ in culture are similar to those in vivo at the time of slice preparation, and thus our observations may be considered good indicators of the normal developmental progress. Consideration of possible implications of this assumption is left for Discussion.

We begin with a general description of the laminar arrange- ment of developing axonal arbors, followed by a more detailed assessment of axonal arbors growing within the "incorrect" target layer, layer 4, and finally a detailed analysis of axonal growth within layer $2 / 3$.

\section{P14}

At the earliest age examined, pyramidal cells in layer $2 / 3$ had a very simple descending axon emerging from the base of the cell body. This main axon followed a straight course down to the subplate, crossing layers $2 / 3$ and 4 mostly unbranched, and forming only one or two branches in layers 5 and/or 6 (Fig. $2 A$ ). These collateral branches also followed descending trajectories, suggesting that they might have been formed by bifurcation of the main axon. On average, each cell made $0.76 \pm 0.17$ collaterals in layer 5 (mean \pm SEM; $n=25$ cells) and $0.72 \pm 0.17$ in layer 6 (see Fig. $4 A$ ). Typically, large growth cones tipped the end of the main axon as well as all collateral branches.

\section{P18}

Four days later in development, layer $2 / 3$ pyramids showed a few additional axonal branches within the cortical layers, which were mostly primary collaterals emerging at a right angle from the main descending axon; this suggests that such branches were formed by interstitial branching. Figure $2 B$ illustrates typical patterns of axonal arborization of layer $2 / 3$ pyramidal neurons at P18. The newly formed collateral branches already extended for tens to hundreds of micrometers in length, following horizontal courses parallel to the cortical layering, and were tipped with growth cones in most cases. Surprisingly, the new axonal branches that developed between P14 and P18 had been formed preferentially in layers 4 and 5 (1.54 \pm 0.27 and $1.58 \pm 0.26$ branches per cell, respectively; $n=26$ cells) and only to a lesser extent in layer $2 / 3(0.69 \pm 0.20$ branches per cell) (see Fig. $4 A)$. There was no increase in layer $6(0.65 \pm 0.15$ branches per cell). This differs from the adult pattern of vertical connections, where 


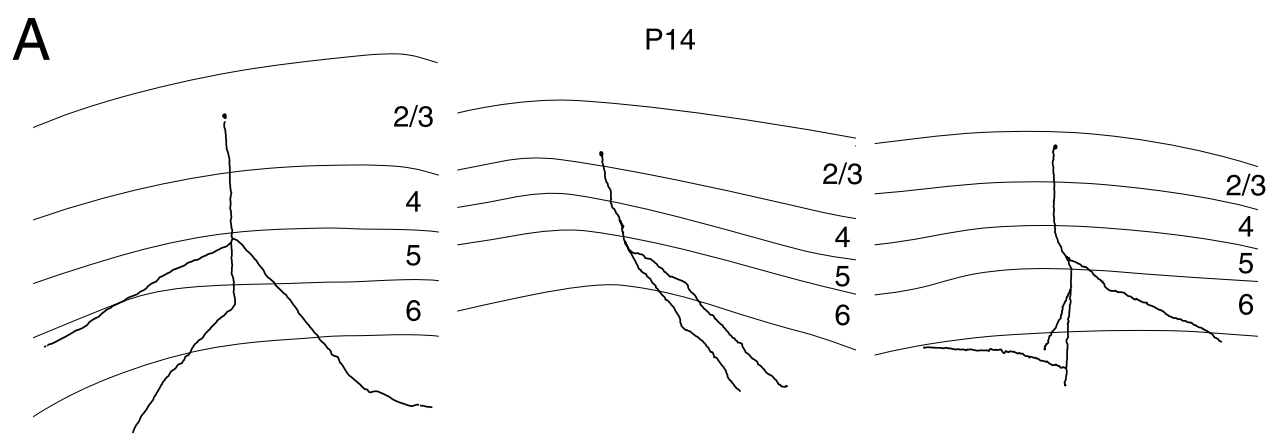

B
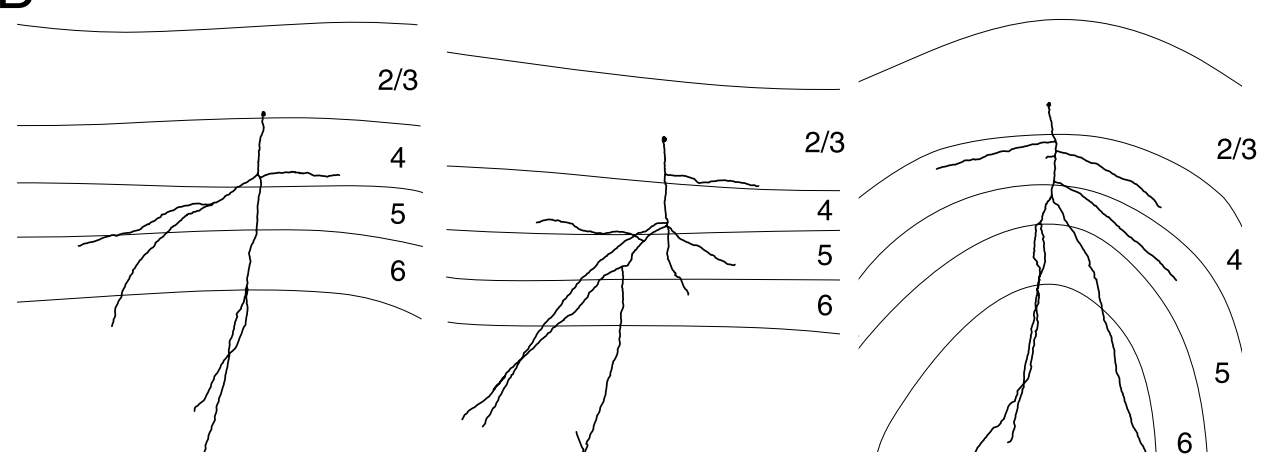

C
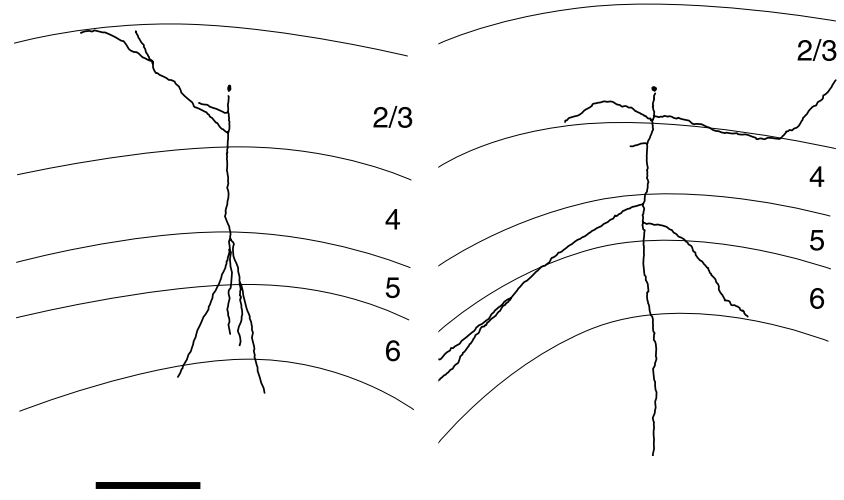

layer $2 / 3$ pyramids branch almost exclusively in layers $2 / 3$ and 5 , and very rarely in layer 4 (Gilbert and Kelly, 1975; Lund and Boothe, 1975) (for review, see Callaway, 1998b) (see also results from older ferrets, below).

\section{$P 22$}

By P22, the extent of axonal arborization had increased specifically in layers $2 / 3(1.69 \pm 0.26$ branches per cell $)$ and $5(2.41 \pm$ 0.40 branches per cell; $n=32$ cells) (see Fig. $4 A$ ). Axon collaterals in layer $2 / 3$ were mostly of primary order, arising from the main axon, and extended horizontally and unbranched within layer $2 / 3$ (Fig. 2C). The length of these branches was variable, ranging from tens to hundreds of micrometers. The increase in number of branch points in layer 5 was attributable partly to the addition of primary collaterals but also to the formation of second and third order branches in some cases ( 9 of 32 cells; data not shown). Axon collaterals that originated in layer 5 remained

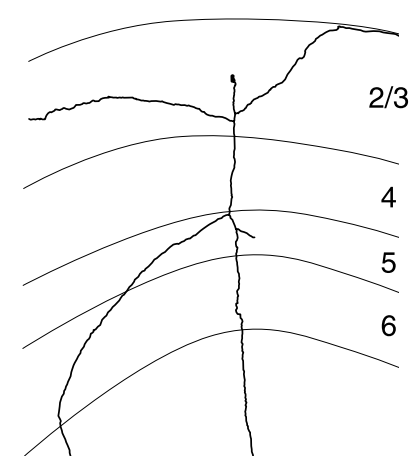

Figure 2. Neurolucida reconstructions of axonal arbors of pyramidal neurons located in layer $2 / 3$ of area 17 slices. Cells developed in vivo and were labeled by expression of GFP in slices from $\mathrm{P} 14(A), \mathrm{P} 18(B)$, and $\mathrm{P} 22(C)$ animals (see Results). $A$, At the earliest age examined (P14), cells had a main descending axon with very few branches, and these were formed in layers 5 and/or 6. B, At $P 18$, cells had formed new branches in layers 4 and 5 , and occasionally also in layer $2 / 3$. Branches in layer 4 were long, and in most cases originated in the vicinity of the layer $4 / 5$ border, although some cells also displayed branches emerging from the center of layer 4. C, By $P 22$, cells displayed axonal branches mostly in layers $2 / 3$ and 5 , where they extended for several hundred micrometers. Branches were rarely found in layer 4 at this stage. Cell bodies are indicated by solid polygons. Laminar boundaries are represented with lines, and cortical layers are indicated by numbers on the side of reconstructions. Scale bar, $300 \mu \mathrm{m}$. mostly in the same layer, extending horizontally and not vertically (Fig. 2C).

In contrast to the overall increase in arborization in layers $2 / 3$ and 5 between P18 and P22, in layer 4 there was a significant decrease in the average number of axonal branches $(0.44 \pm 0.13$ branches per cell at $\mathrm{P} 22$ versus $1.54 \pm 0.27$ at $\mathrm{P} 18 ; p<0.05$ ) (see Fig. $4 A$ ). In addition, the collaterals that remained in layer 4 were either very short $(<50 \mu \mathrm{m})$ (Fig. $2 C$ ) or grew unbranched directly away from layer 4 toward one of the neighboring layers $(2 / 3$ or 5$)$. This is consistent with the notion that layer 4 is not a layer of preference for the growth or branching of axon collaterals by layer $2 / 3$ pyramids. These results also suggest that a significant number of branches that had been formed previously in layer 4 may now have been eliminated by selective pruning. Below, we will further explore these changes on the basis of a more detailed analysis of the distribution of axonal branches within the depth of layer 4 . 
A

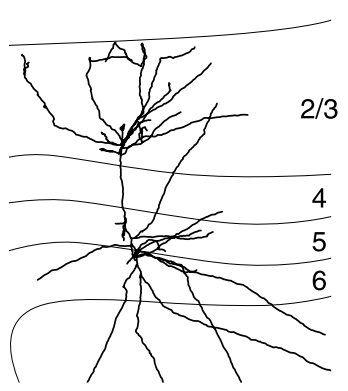

B

Figure 3. Reconstructions of axonal arbors of layer $2 / 3$ pyramidal neurons that developed in vivo and were labeled with GFP in area 17 slices, obtained from $\mathrm{P} 26(A)$ and $\mathrm{P} 30(B)$ ferrets. These cells all have a main descending axon extending down to the white matter and numerous collateral branches in layers $2 / 3$ and 5 that extend laterally for several hundred micrometers. Most of the primary axon collaterals also give off secondary branches. These cells contain no axonal branches in layer 4. Conventions are as in Figure 2. Scale bar, $300 \mu \mathrm{m}$.
P26

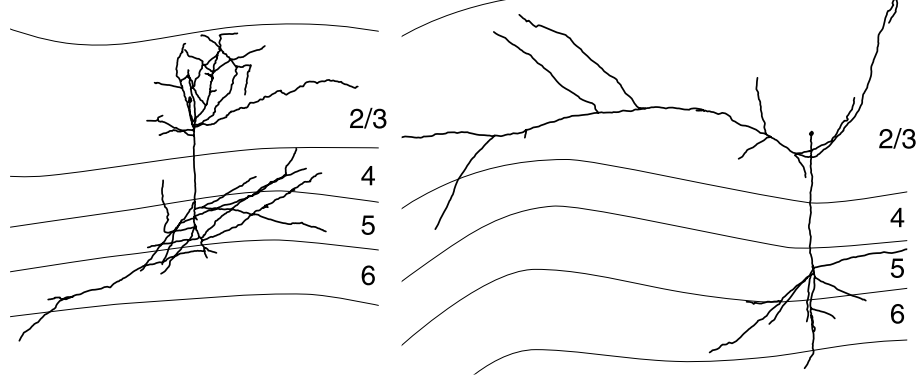

P30

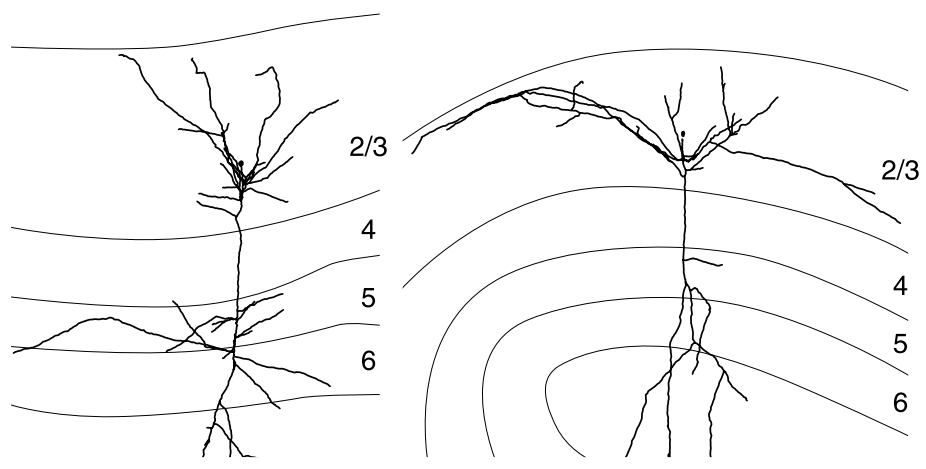

Finally, the number of branch points per cell observed in layer 6 remained low $(0.63 \pm 0.15)$, similar to that at $\mathrm{P} 14$ and P18 (Figs. 2, $4 A$ ).

\section{P26}

Between P22 and P26 there was a large, layer-specific increase in axonal branching and growth, so that at this stage layer $2 / 3$ pyramids displayed well elaborated axonal arbors in layers $2 / 3$ and 5. As illustrated in the examples of Figure $3 A$, during this period there occurred a significant increase in the number of primary as well as secondary and tertiary branches in both layers $2 / 3$ and 5 (from $1.69 \pm 0.26$ and $2.41 \pm 0.40$ branch points per cell, to $10.85 \pm 1.01$ and $8.73 \pm 1.02$, respectively; $n=26$ cells) (Fig. $4 A$ ). It is interesting to note that the majority of axon collaterals originating in each of these layers remained in that same layer, without growing into or across layer 4 (Fig. $3 A$ ). We also observed a significant increase in the density of axonal arborization in layer 6 , but up to a smaller absolute value $(3.27 \pm 0.56)$ than in layers $2 / 3$ or 5 (Fig. $4 A$ ). Layer $2 / 3$ pyramidal cells at this stage displayed a slight increase in the number of axonal branches in layer 4 with respect to the earlier stage examined (from $0.44 \pm 0.13$ to $1.00 \pm$ $0.27)$. However, this change was not significant $(p>0.05)$ and was much smaller than that observed for any other layer. In addition, the decrease in the number of branches in layer 4 observed between P26 and P18 was not statistically significant $(p>0.05)$. These "ectopic" branches observed in layer 4 were always short or growing directly toward layers $2 / 3$ or 5 , as already observed at P22. The analysis of the positions of these branches within layer 4 (see below) also shows that these branches were found preferentially at the edges of layer 4 .

\section{P30}

The density of axonal branching continued to increase in layer $2 / 3$ until P30 (the last age examined), although the change with respect to $\mathrm{P} 26(16.36 \pm 1.38$ compared with $10.85 \pm 1.01$ branch points per cell; $n=25$ cells) was less than what occurred between P22 and P26 (see above) (Fig. $4 A$ ). Figure $3 B$ shows some examples of axonal arbors from layer $2 / 3$ pyramids at this age. Interestingly, new primary branches were still added to the main axon within layer $2 / 3$, in addition to second and third order collaterals, thus further contributing to the elaboration of the axonal arbor (see below). In contrast, there were no significant changes in branching density in either layer 5 or layer 6 with respect to the previous age examined, and as before, collaterals were primary as well as secondary, and even tertiary (Figs. 3B, 4A). Finally, the number of axonal branches in layer 4 at this age was slightly, but not significantly, reduced with respect to P26 $(0.6 \pm 0.31$ branches per cell). There was, however, a significant reduction in axonal branches found in the center of layer 4 (see below). In addition, the total number of branches in layer 4 at P30 was significantly smaller compared with P18 $(p<0.05)$.

\section{Ectopic axonal branches from layer 2/3 pyramids are transiently present in layer 4}

Previous studies have shown that in the adult mammalian cerebral cortex, layer 2/3 pyramidal neurons very rarely make any axonal branches in layer 4 (Gilbert and Kelly, 1975; Lund and Boothe, 1975; Tigges and Tigges, 1982; Katz, 1991; Burkhalter et al., 1993; Callaway and Wiser, 1996; Callaway, 1998a) (for review, see Callaway 1998b). However, as described above, we found that in area 17 of the ferret primary visual cortex, layer $2 / 3$ pyramids 


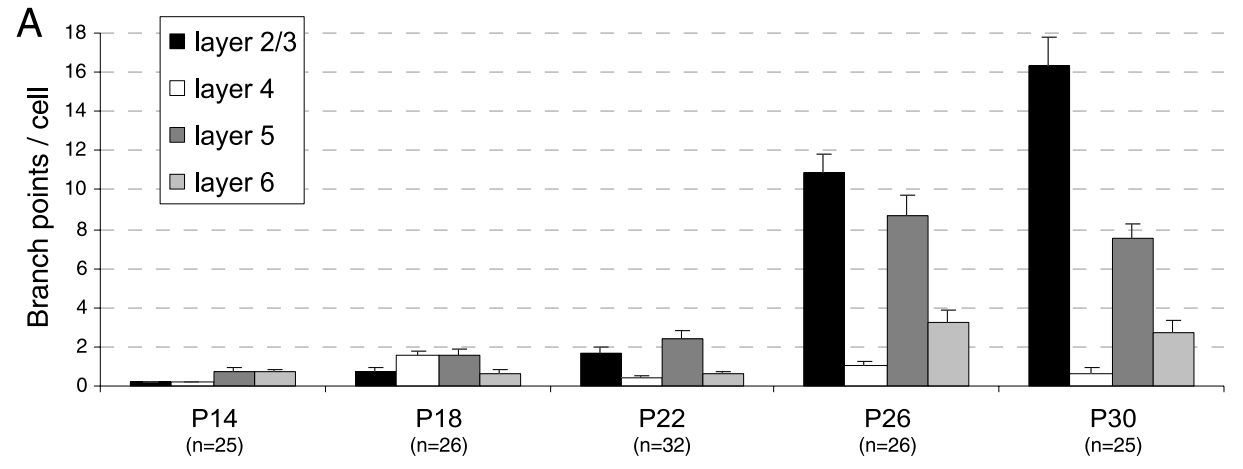

B Axonal branches in layer 4 (P18)

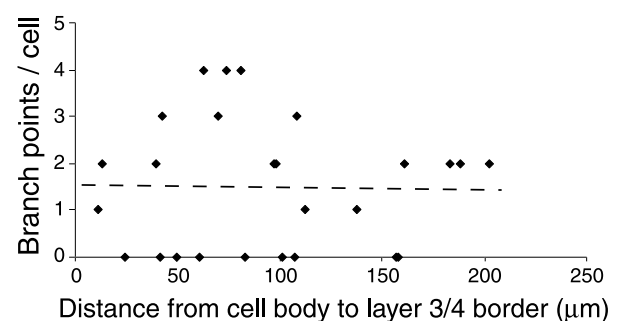

C Axonal branches in layer 4

D

Axonal branches in layer 4 (P30)

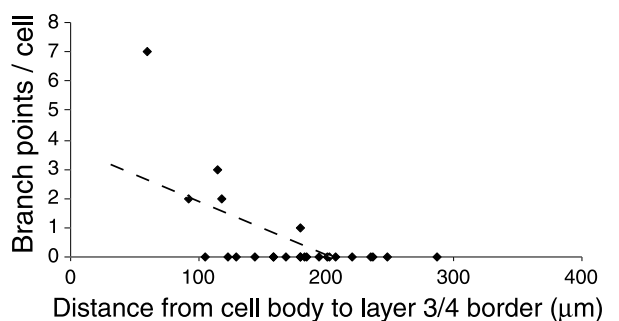

F

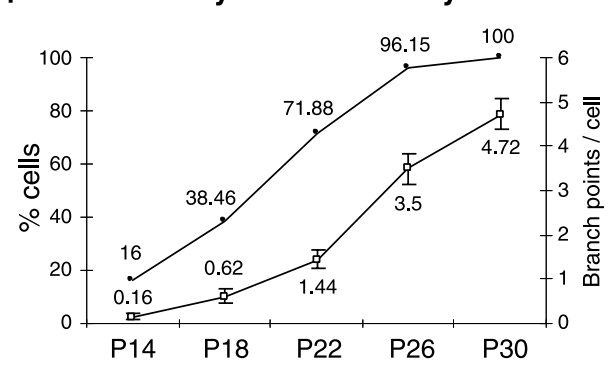

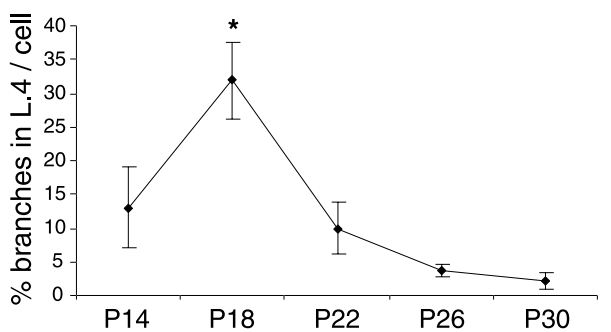

E Axonal branches in layer 4 (P18)

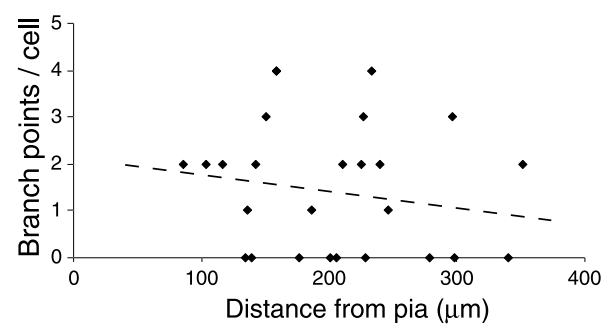

G $\%$ Axonal branch types in layer $2 / 3$

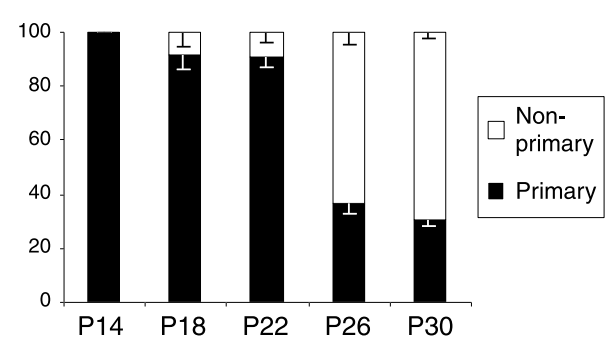

Figure 4. Quantitative analysis of the axonal arborizations of layer $2 / 3$ pyramids that developed in vivo and were labeled in area 17 slices obtained from animals of various postnatal ages. $A$, Histograms showing the mean $( \pm$ SEM) numbers of axonal branch points per cell in each cortical layer at the different ages. Postnatal ages are indicated below each group of bins, and the number of cells analyzed in each case is in parentheses. Bins corresponding to different layers have different fill patterns, as indicated above. Layer $2 / 3$ pyramidal neurons from P14 slices had very simple axonal arbors, making branches almost exclusively in layers 5 and 6 . Between P14 and P18 the number of axonal branches in layer 4 increased significantly, and pyramidal cells displayed branches preferentially in layers 4 and 5. By P22 the number of branches in layer 4 was significantly reduced, and increased preferentially in layers $2 / 3$ and 5 . Between P22 and P26 there was a dramatic increase in the numbers of axonal branches in layers $2 / 3$ and 5 , whereas they remained small in layer 4. The number of axonal branches in layer $2 / 3$ continued to increase until P30. $B, D, E$, Scatter plots of the number of axonal branch points in layer 4 of each cell with respect to the distance from the cell body to the layer $3 / 4$ border $(B, D)$ or to the pial surface $(E)$ at $\mathrm{P} 18(B, E)$ and $\mathrm{P} 30(D)$. Dashed lines represent the regression line for the plotted values. The relatively uniform distributions of points in $B$ and $E$ illustrate that at P18 the number of branches in layer 4 is not correlated with the proximity of the cell body to layer $4(B$; $\left.r^{2}=0.0002\right)$ or to the pia $\left(E ; r^{2}=0.022\right)$. Only at P30 was there some correlation between the number of branches in layer 4 and the distance from cell body to layer $3 / 4$ border $\left(D ; r^{2}=0.38\right)$. At this age, this reflects the proximity to the layer $3 / 4$ border of the 5 of 25 cells that displayed any branches in layer 4. $C$, Plot showing the mean $( \pm$ SEM $)$ percentage of axonal branches in layer 4 per cell. Cells at P18 had $31.8 \pm 5.8 \%$ of their axonal branches within layer 4 , a proportion significantly larger $(*)$ than at any other age examined. Differences were considered statistically significant for $p<0.05$ ( $t$ test). $F$, Plot showing the percentage of cells containing primary axon collaterals within layer $2 / 3$ and the mean $( \pm$ SEM $)$ numbers of primary

axonal branches per cell at the different postnatal ages. Mean values at each age are indicated. At P14, only 16\% of cells had formed primary axon branches in layer 2/3, but the percentage increased gradually until P26, when primary branches were present in 25 of 26 cells (96\%). Accordingly, the number of primary axon collaterals within layer $2 / 3$ per cell also increased significantly during the period analyzed. This increase was slow from P14 to P22 and then much faster after P22. $G$, Histogram illustrating the proportion of primary versus nonprimary (secondary, tertiary, etc.) axonal branches within layer $2 / 3$ at the postnatal ages analyzed (mean \pm SEM). Primary branches and nonprimary branches are indicated by black and white, as indicated. Between P14 and P22, virtually all branches originated from the main axon (primary branches), whereas at P26 and P30 only 30-35\% of branches were primary.

display a significant percentage of axonal branches in layer 4 early in development. As shown in Figure $4 C$, at P18 almost $32 \%$ of the axonal branches made by these cells were ectopically located in layer 4. However, this ratio quickly decreased to $10 \%$ at P22 and was reduced still further at subsequent stages. Indeed, from P22 onward, layer $2 / 3$ pyramids made axonal branches with a clear preference for layers $2 / 3$ and 5 , as shown in Figure $4 A$, in accordance with current knowledge of adult mammalian local cortical circuitry (for review, see Callaway, 1998b).
These findings raise three main questions. Is there a particular kind of layer $2 / 3$ pyramid that makes ectopic branches in layer 4 at P18? Are the incorrect branches found at particular depths within layer 4? How are these collaterals "eliminated" later in development? In 1991, Katz described that during the development of vertical connections from layer $2 / 3$ pyramidal neurons in cat striate cortex, axonal branches were incorrectly made in layer 4 only by pyramids located close to the layer $3 / 4$ border. He then showed that in such cases this was caused by the short distance 
between the cell body and layer 4, which forced those cells to initiate primary axon collaterals in the immediately underlying layer.

To test whether this was the reason for the occurrence of axonal branches in layer 4 in our sample, we related the number of axonal branches made in layer 4 by each cell to the distance of its cell body from the layer $3 / 4$ border. As shown in Figure $4 B$, at P18 the number of branches made in layer 4 had no correlation $\left(r^{2}=0.0002\right)$ with the distance between the cell body and the layer 3/4 border. At P30, however, when most cells lacked axonal branches in layer 4 , the cells with layer 4 branches were located close to the layer 3/layer 4 border (Fig. 4D). Indeed, from P18 to P30 we observed a progressive increase in the correlation between branches in layer 4 and the distance of the cell from the layer 3/layer 4 border. The number of branch points detected within layer 4 was inversely proportional to the distance from cell body to layer 4, in agreement with previous observations (Katz, 1991). This correlation was highest at the latest stage examined (P30), with a correlation coefficient of $r^{2}=0.38$ (Fig. 4D).

Given that layer $2 / 3$ is formed by the successive apposition of neuroblasts during a long period of time in the ferret (between P14 and P22) (Jackson et al., 1989), another possible explanation could be that the branches observed in layer 4 at P18 are "mistakes" transiently displayed by only the least mature neurons within layer $2 / 3$. Taking into consideration that in the cerebral cortex less mature neurons are positioned more superficially than more mature ones (Jackson et al., 1989), we tested this second hypothesis by relating the number of axonal branches made in layer 4 by each pyramid to the proximity of the cell body to the pial surface. As shown in Figure $4 E$, at P18 there was no significant correlation $\left(r^{2}=0.022\right)$ between the formation of ectopic axonal branches in layer 4 and the maturity/position of neurons in layer $2 / 3$.

To better understand the mechanisms by which the number of branches in layer 4 is reduced in the late stages of visual cortical development, we analyzed developmental changes in the spatial distribution of axonal branch points within layer 4. At P14 very few axonal branches were made in layer 4 , and these were located mostly in the lower half of this layer (closer to layer 5) (Fig. 5A). At P18, branches appeared homogeneously distributed along the depth of layer $4(0.15-0.20$ branches per cell on average within each fifth of the layer) except for the lower fifth, closest to layer 5 , where the number of branches was four times greater than at other depths $(0.84 \pm 0.18$ branches per cell) (Fig. $5 B)$. In contrast, at P22 the distribution of branches was again relatively homogeneous, including the portion closest to layer 5 (Fig. 5C). When the same type of analysis was performed within layer 5, it showed that at P18 the frequency of axonal branches was highest closer to layer 4, and it decreased progressively from there toward the layer $5 / 6$ border (Fig. $5 F$ ). At P22 the distribution in layer 5 was very similar to that at P18, but the overall frequency was higher than at the previous stage (Fig. 5G). At P26 and P30, axonal branches in layer 4 occurred mostly in the top- and bottom-most edges, and thus in the vicinity of the borders with the neighboring layers (Fig. 5D,E).

On the basis of these results, it might be suggested that axonal branches at the edges of layer 4 shift into other layers during development. Therefore we were particularly interested in determining whether there is also a decrease in the number of branches in the center of layer 4 . Such a loss most likely reflects elimination of incorrect branches. The number of branch points observed in the center of layer 4 (central 60\%) (Fig. 5H) was significantly
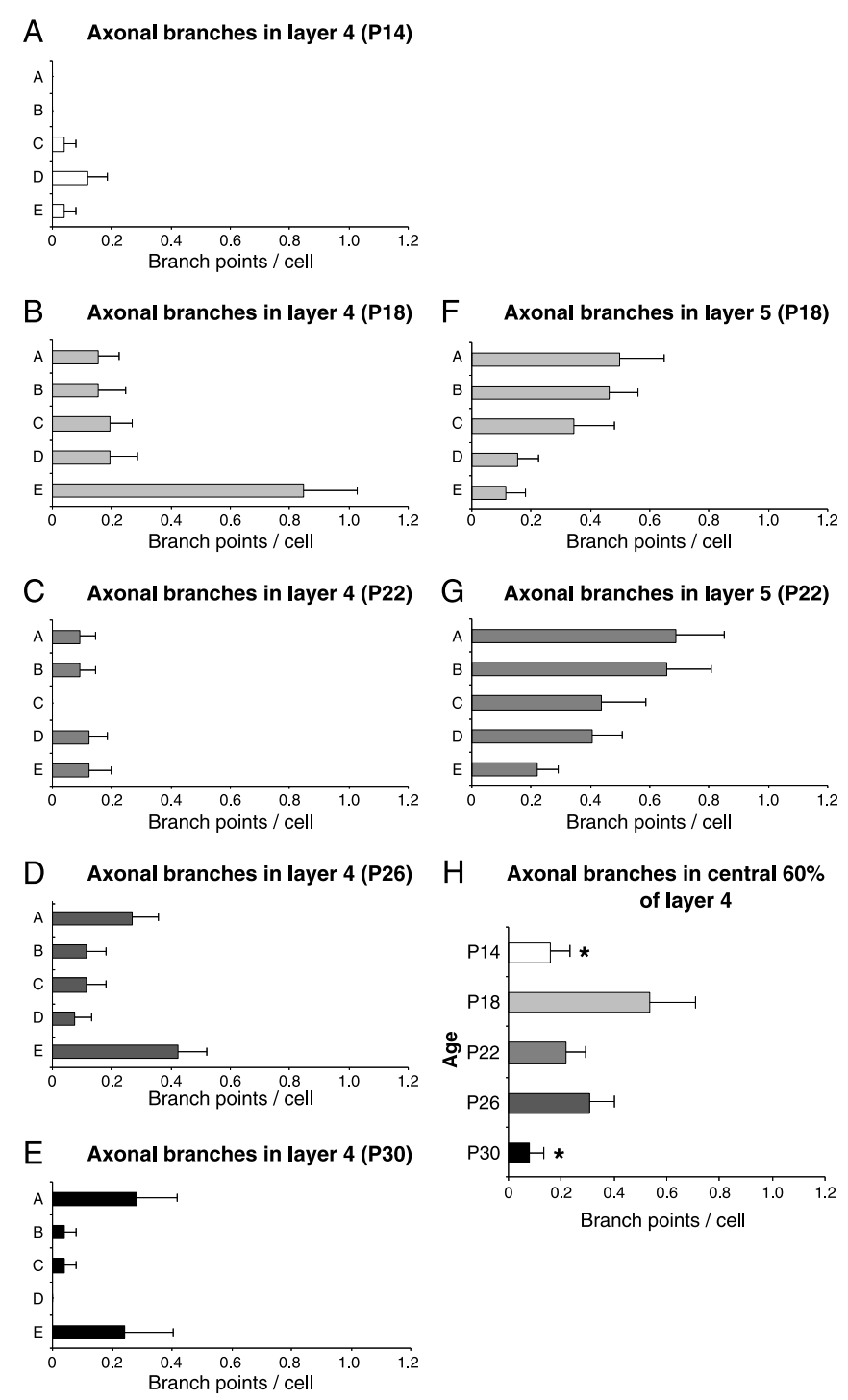

Figure 5. Quantitative analysis of the distribution of axonal branch points within layers 4 and 5 at various postnatal ages. $A-G$, Histograms showing the mean $( \pm$ SEM) numbers of axonal branch points per cell found within subdivisions of layer $4(A-E)$ and layer $5(F, G)$. Each bin corresponds to $20 \%$ of the depth of the layer, and the bins are labeled $\mathrm{A}$ through $\mathrm{E}$ from the top to the bottom of the layer. A, At P14 a small number of axonal branches were made only in the lower $60 \%$ of layer 4 . $B$, At P18 axonal branches were most abundant in the deepest fifth of layer 4 (bin E), although they were also present more superficially in layer 4. $C$, By P22 the overall number of axonal branches in layer 4 had decreased, particularly in the bottom fifth (bin E). At P26 and P30 $(D, E)$, the few axonal branches that remained in layer 4 were mainly at the top and bottom edges of the layer (bins A and E). F, $G$, The distribution of axonal branches in layer 5 remained similar between P18 and P22, with branches occurring most frequently in the top half of the layer (bins $\mathrm{A}-\mathrm{C}$ ). $H$, Histogram showing the mean $( \pm$ SEM) numbers of axonal branch points per cell observed in the central $60 \%$ of layer 4 (bins B-D combined) at the various developmental stages analyzed. The frequency of branch points per cell in this central region increased significantly between P14 and P18 and showed a progressive reduction at later ages. The reduction was significant by P30 $\left({ }^{*} p<0.05\right)$. Sample sizes are as in Figure 4.

reduced by almost sevenfold between P18 (0.54 \pm 0.17 branches per cell) and P30 (0.08 \pm 0.06 branches per cell) (Fig. $5 H)$.

Thus, at both P18 and P22, layer 2/3 pyramids show a clear preference for axonal branching in the vicinity of the layer $4 / 5$ border, which probably leads to the occurrence of many ectopic 
A
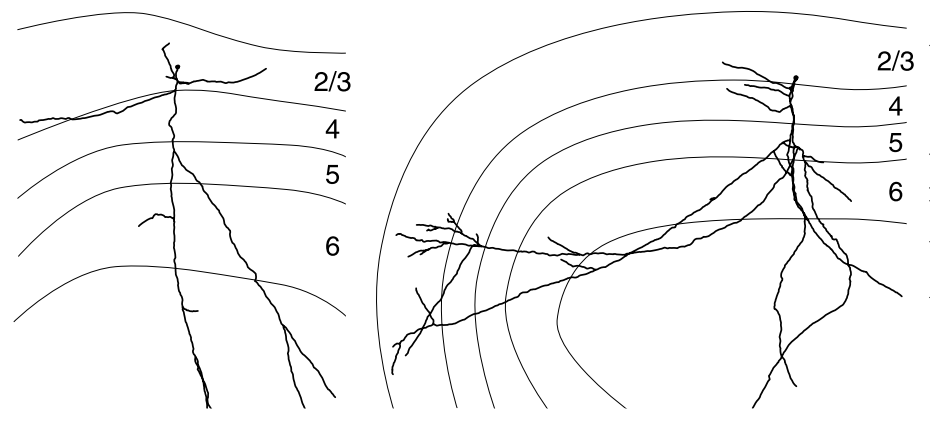

P14 + 5 DIV$$
\text { B }
$$
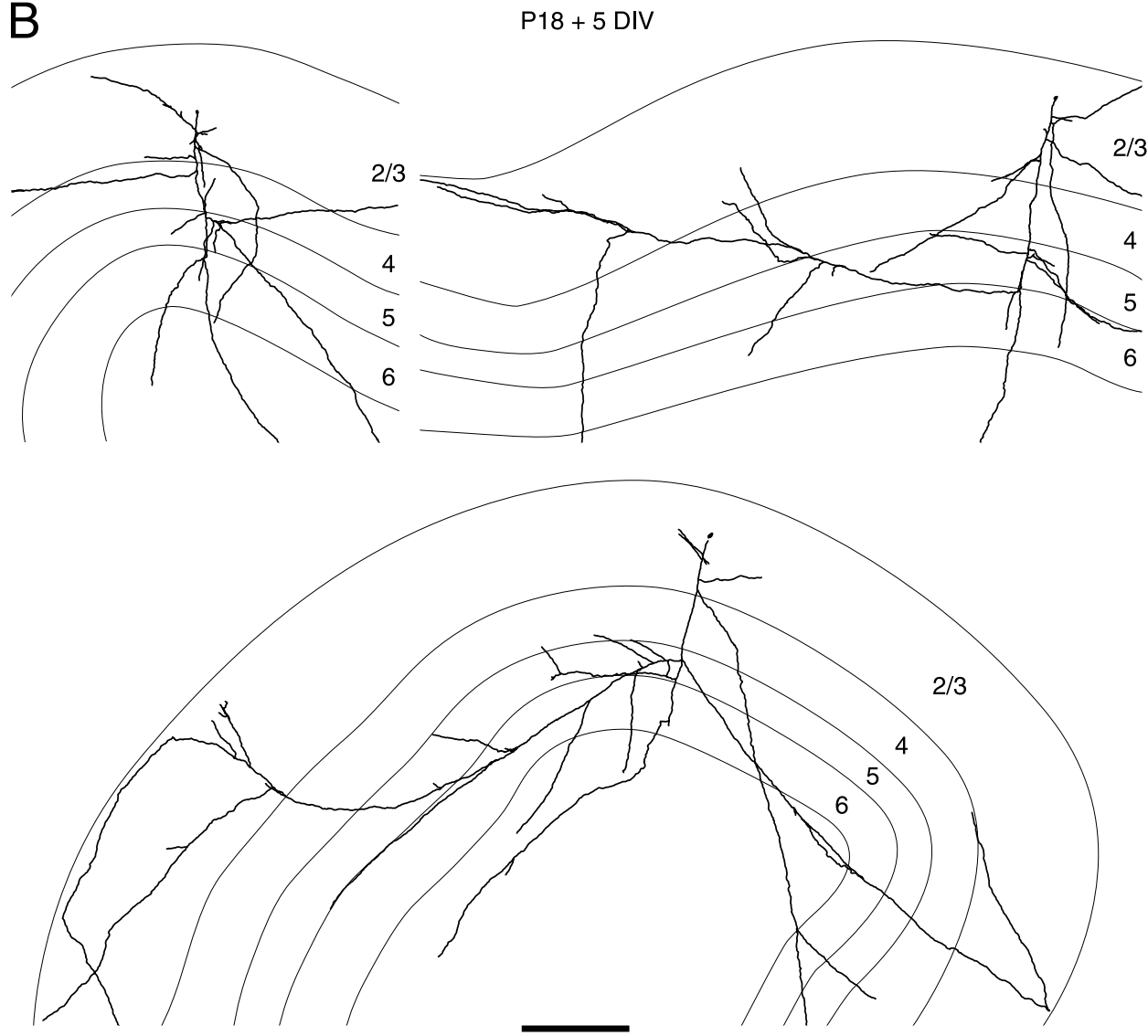

$2 / 3$

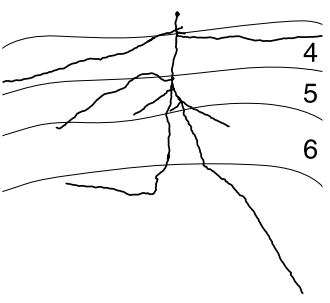

$/ 3$ 
Figure 7. Examples of axonal arbors of layer $2 / 3$ pyramidal neurons in area 17 slices obtained from P22 animals and allowed to develop in vitro for $5 \mathrm{~d}$ $(P 22+5 D I V)$. Under these conditions, pyramids developed complex axonal arbors extending considerable distances and branching repeatedly but almost exclusively in layers $2 / 3$ and 5 . In many cases collaterals grew from deep layers into layer $2 / 3$, where they sprouted numerous branches while avoiding layer 4. Conventions are as in Figure 2. Scale bar, $400 \mu \mathrm{m}$.

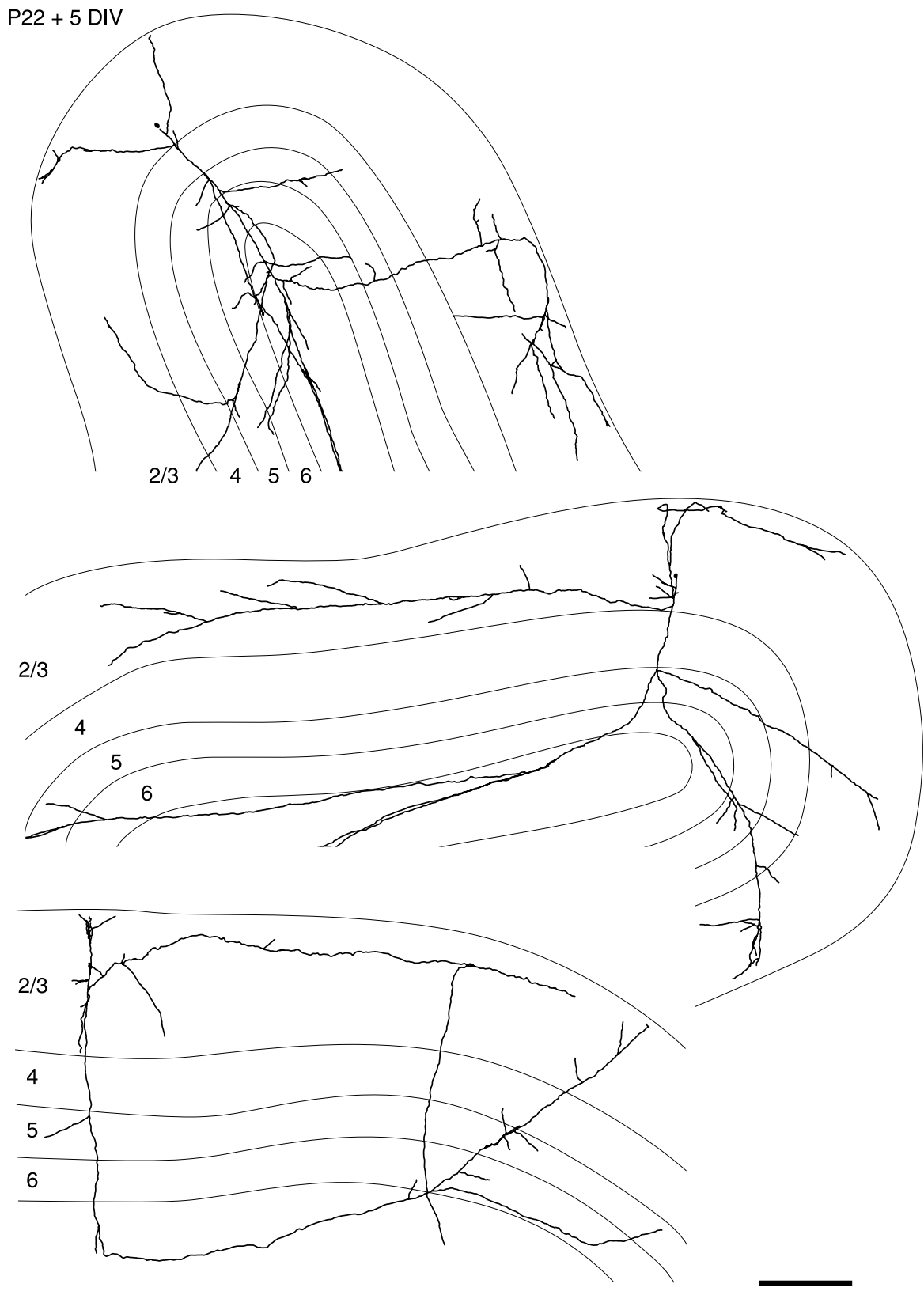

In conclusion, the slow but significant increase in the number of axonal branches observed in layer 2/3 from P14 to P22 (Fig. 4A) was caused mainly by an increase in the number of primary collaterals per cell. From P22 through P30, the number of primary collaterals continued to increase but at a faster rate than before, and by P30 every cell had at least one primary branch in layer 2/3. In addition, from $\mathrm{P} 22$ to $\mathrm{P} 30$ there was a global burst of branching activity in layer $2 / 3$ pyramids, involving the massive formation of collaterals in layers $2 / 3,5$, and 6 . In layer $2 / 3$ this was mainly the result of the elaboration of primary collaterals into secondary and tertiary branches.

\section{Development of laminar specificity in vitro depends on the age of the animal when slices are made}

In an earlier study, we reported that layer $2 / 3$ pyramidal cells from P14/P15 ferret visual cortex fail to form layer-specific axonal branches during 5-7 d in culture (Butler et al., 2001). Thus, it remains unknown whether cultured layer $2 / 3$ pyramids are unable to develop layer-specific axonal branches in the absence of extrinsic influences, or if the lack of specificity reflects a recapitulation of the normal developmental program. We therefore investigated whether the lack of specific development in vitro might reflect the immaturity of the tissue at the time the slices were prepared $(\mathrm{P} 14 / \mathrm{P} 15)$ rather than a lack of preservation of normal cues or extrinsic influences in the slice cultures. We made organotypic slice cultures of area 17 from P14, P18, and P22 ferrets and maintained them in culture for $5 \mathrm{~d}$. In agreement with previous descriptions (Butler et al., 2001), only a few pyramidal cells at P14 +5 DIV developed axonal branches specifically in layers $2 / 3$ and 5 ( 5 of 20 cells), whereas the majority of cells made branches also in layer 4 (Fig. $6 A$ ). On average, layer $2 / 3$ pyramids did not exhibit a preference for branch formation in layers $2 / 3$ and 5, and the number of axonal branch points displayed among all cortical layers was statistically homogeneous $(p>$ 0.05) (see Fig. 8A). 
In contrast, when P18 cortical slices were maintained in culture for 5 DIV, axonal arbors developed branches with a significant preference for layers $2 / 3$ and 5 and had 2- to 3.5-fold more branches in these layers than in layers 4 or 6 (Figs. $6 B, 8 B$ ). Branches already present in layer 4 when slices were prepared at P18 (1.54 \pm 0.27 branch points per cell; values from $24 \mathrm{hr}$ cultures) (Fig. 4A) were apparently not eliminated after $5 \mathrm{~d}$ in vitro (2.25 \pm 0.58 branches per cell) (see Fig. $8 B$ ) as they would be in vivo (compare with $\mathrm{P} 22: 0.44 \pm 0.13$ branches per cell; values from $24 \mathrm{hr}$ cultures) (Fig. $4 A$ ). Finally, layer $2 / 3$ pyramids from P22 slices continued to make axonal branches preferentially in layers $2 / 3$ and 5 during $5 \mathrm{~d}$ in culture (Figs. 7, 8C).

It is interesting to note that in all slices cultured for $5 \mathrm{DIV}$, the in vitro growth closely paralleled the growth that would have occurred in vivo (compare Figs. $4 A$ and 8). For example, the numbers of branches in layer $2 / 3$ at P14, P18, and P22 + 5 DIV consistently increased, with values of $5.55 \pm 0.88,8.05 \pm 1.14$, and $14.15 \pm 1.60$ branches per cell, respectively (Fig. 8). This is similar to the increases occurring in vivo as evidenced by our observations in slices at P18, P22, and P26 with only $24 \mathrm{hr}$ in culture (Fig. 4A).

\section{Development of apical and basal dendritic arbors in vivo}

After finding that layer $2 / 3$ pyramids exhibit a burst of axonal elaboration between P22 and P26, we decided to analyze the development of the apical and basal dendritic arbors, with special attention to the occurrence of a similar period of increase in branching activity. The apical dendritic arbors displayed a steady but gradual increase in size and complexity from P14 to P30 (Fig. 9). The number of apical dendritic branches increased from $6.5 \pm$ 0.6 branches per cell at P14 to $19.2 \pm 1.2$ branches per cell at P30 (values from $24 \mathrm{hr}$ cultures), with a significant increase between P22 and P26 $(p<0.05)$ (Table 1$)$. The total length of the apical dendritic arbor increased accordingly (Table 1).

At P14 the basal dendrites were very simple, short, and mainly unbranched $(80.8 \pm 15.5 \mu \mathrm{m}$ in length and $0.5 \pm 0.2$ branches per cell; values from $24 \mathrm{hr}$ cultures). Between P14 and P22 some changes were apparent, not so much in the number of basal dendrites but more in their length $(351.2 \pm 29.0 \mu \mathrm{m}$ at P22) and branching ( $3.6 \pm 0.5$ branches per cell at P22) (Table 1$)$. However, between P22 and P26 the degree of elaboration of the basal dendritic arbors changed dramatically (Fig. 9), with a significant increase in the number of basal dendrites (from $3.2 \pm 0.2$ dendrites per cell at P22 to $4.2 \pm 0.2$ at P26), the number of branches per cell (13.9 \pm 1.2 at P26), and total length of arbors (1003.6 \pm $68.5 \mu \mathrm{m}$ at P26; values from $24 \mathrm{hr}$ cultures; $p<0.001$ for all comparisons). Finally, between P26 and P30 the basal dendritic arbors increased in complexity even further (Fig. 9, Table 1).

To quantitatively compare the changes that occurred in apical and basal dendrites during development, we calculated the DGI, a weighted sum of three measures of dendritic complexity: number of dendrites, branch points, and total length [adapted from McAllister et al. (1995); see Materials and Methods]. A DGI value of 1 indicates no significant change in dendritic form, and greater values indicate significant increases in complexity. Figure 10 illustrates the changes in DGI that occurred during development compared with the initial stage analyzed (P14). In agreement with our observations, the value of DGI of the apical dendrite increased gradually during the five ages examined (Fig. $10 \mathrm{~A}$ ), with relative increases in DGI ranging between 0.27 (from $\mathrm{P} 18$ to $\mathrm{P} 22$ ) and 0.89 (from P14 to P18). For basal dendrites, the
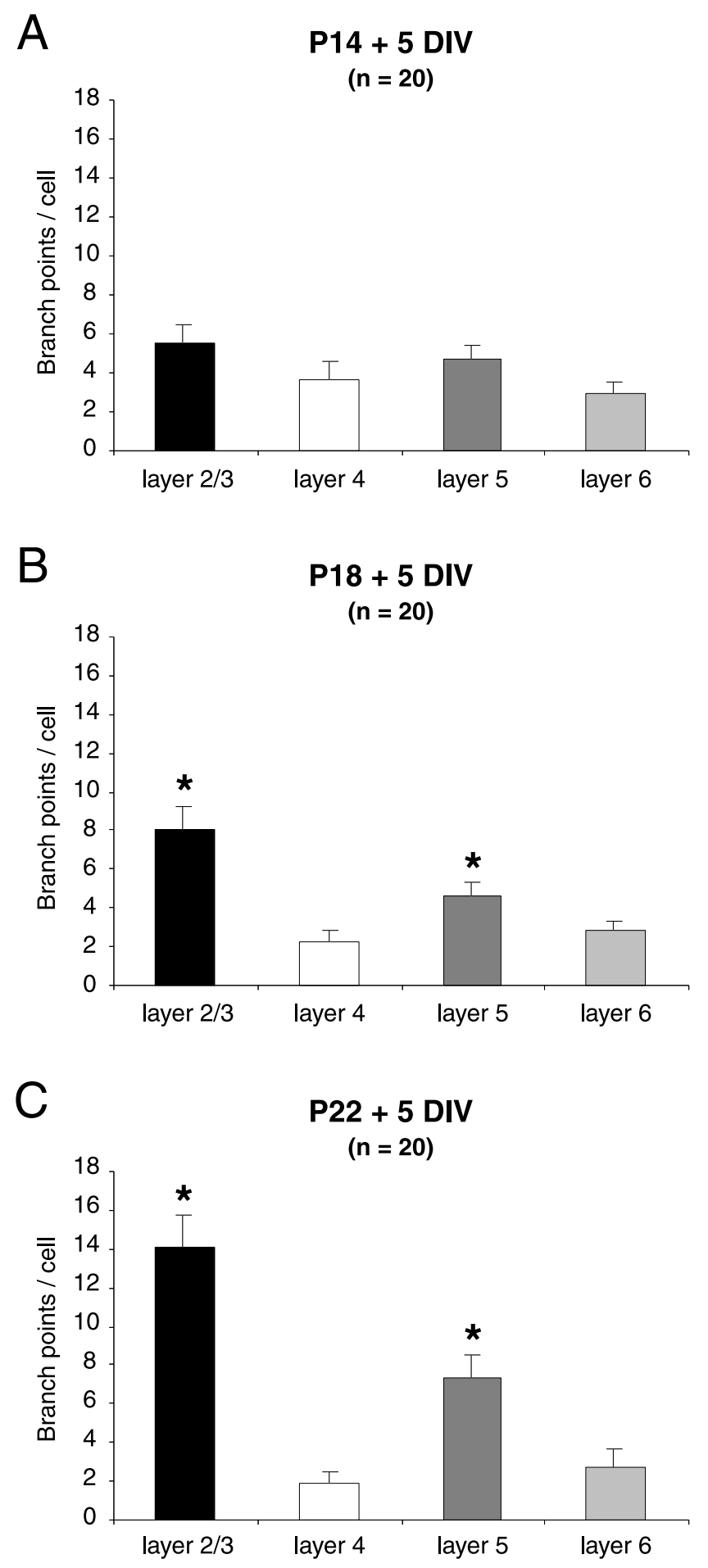

Figure 8. Histograms showing the number of axonal branch points per cell for layer $2 / 3$ pyramidal neurons in area 17 slices that were obtained from animals of various postnatal ages and developed for 5 DIV. $A$, Pyramidal neurons from $\mathrm{P} 14$ slices $(\mathrm{P} 14+5 \mathrm{DIV})$ displayed axonal arbors with numerous branch points but failed to exhibit any specificity for layers $2 / 3$ and 5. In $P 18+5 D I V(B)$ and $P 22+5 D I V(C)$ slices, pyramidal neurons developed axonal arbors with branch points preferentially formed in layers $2 / 3$ and 5 , where their number was significantly larger than in layers 4 and 6 . The number of branches formed in layer $2 / 3$ in P22 + 5 DIV slices was also significantly greater than in the same layer in P18 +5 DIV slices. ${ }^{*} p<0.05$. Conventions are as in Figure 4. 
P14<smiles>CCCC=NCC(C)C</smiles>

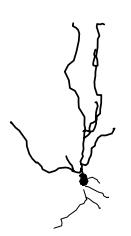
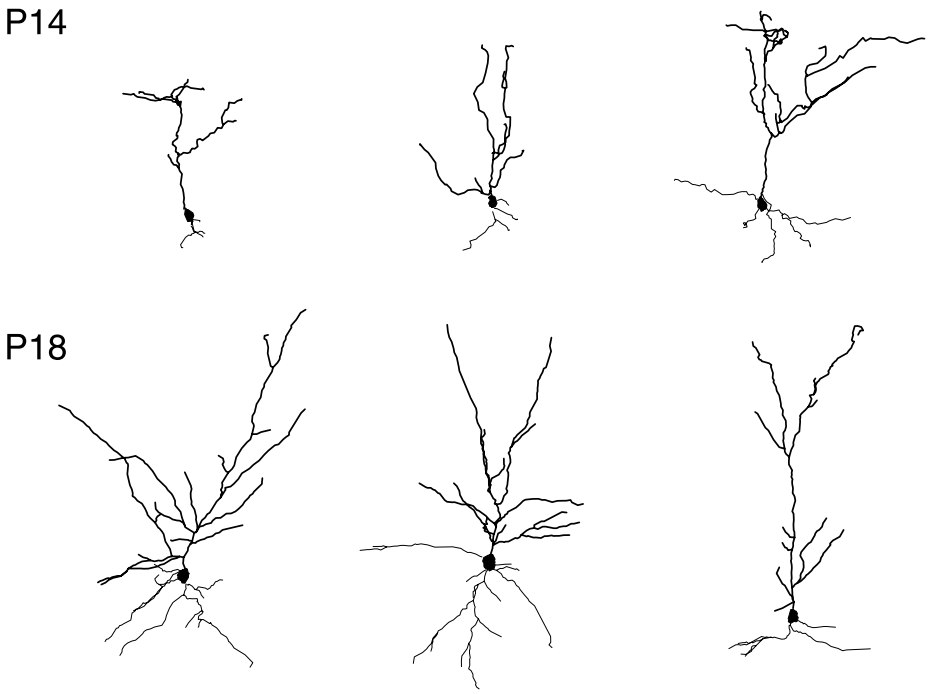

P22
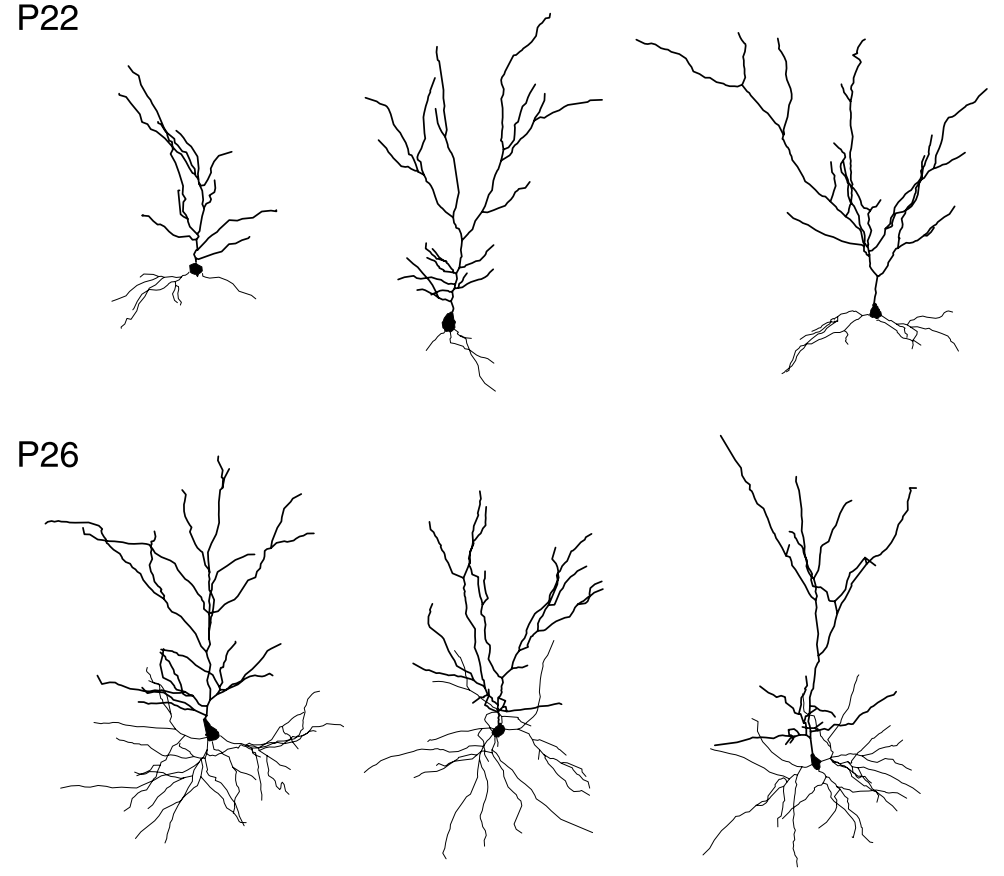

Figure 9. Reconstructions of representative dendritic arbors of layer $2 / 3$ pyramidal neurons that developed in vivo before labeling with GFP for $24 \mathrm{hr}$ in vitro. Slices were obtained from various developmental stages, as indicated. Thick lines represent the apical dendritic arbor, and thin lines represent the basal dendrites. Cell bodies are indicated by solid polygons. Apical dendritic arbors were very simple at $P 14$, with few branches, and were short in total length. As development proceeded, apical dendrites increased progressively in size and complexity until the last age examined. Basal dendrites were short and simple between $P 14$ and $P 22$, showing few branches. Between $P 22$ and $P 26$ the basal dendritic arbor displayed a dramatic increase in complexity, reflective of an increase in the number of primary dendrites and branches, as well as in total length. At P30, basal dendrites remained more extensive and more elaborate than at earlier ages. Scale bar, $100 \mu \mathrm{m}$.

\section{P30}

DGI increased significantly by P18 (3.45), but then it remained relatively similar at P22 (4.11). However, at P26 the DGI increased drastically to 13.47 and still further up to 20.27 at P30 (Fig. 10B).

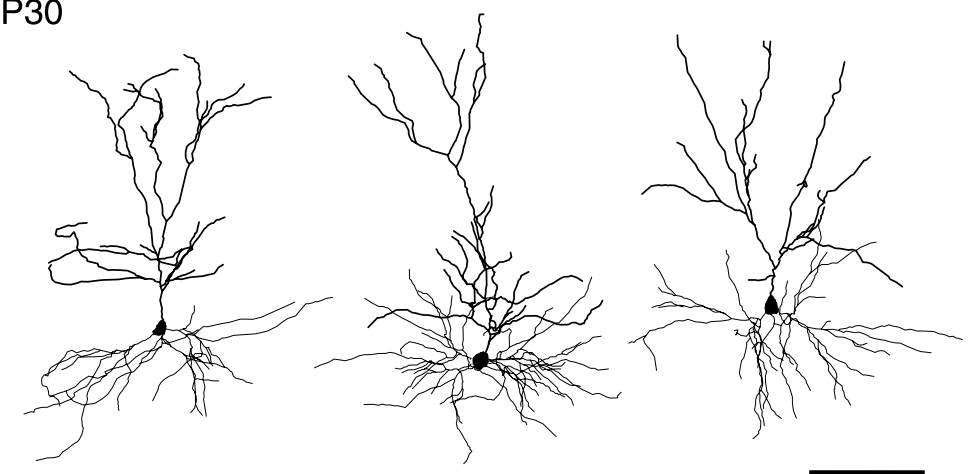

\section{DISCUSSION}

Studies of cat visual cortex (area 17) (Katz, 1991) showed that cortical neurons develop local axonal arbors predominantly in the same layers where they are present in the adult, without forming 
Table 1. Values of three measures of dendritic growth during development

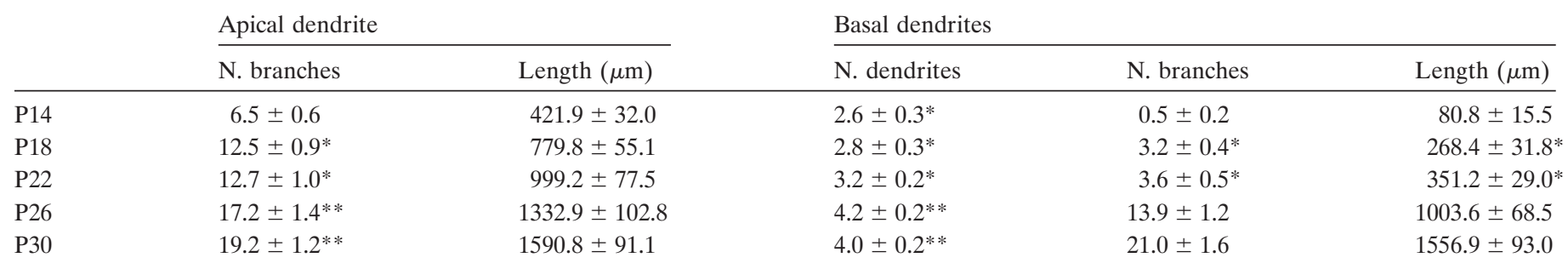

Mean \pm SEM of measurements from apical and basal dendrites of layer 2/3 pyramidal neurons that developed in vivo at each postnatal age. The sample size per group is P14, $n=25, \mathrm{P} 18, n=26, \mathrm{P} 22, n=32, \mathrm{P} 26, n=26$, and $\mathrm{P} 30, n=25$. Single and double asterisks indicate statistically homogeneous groups at the level of $p<0.05$. All other comparisons of the same measures between different ages are significantly different. Statistical significance was calculated by single-factor ANOVA and confirmed by pair-wise $t$ tests. N. branches, Number of branches; N. dendrites, number of dendrites; Length, total dendritic length.

extensive collaterals in other layers. This has been confirmed for cells in other layers and in other species (Callaway and Katz, 1992; Burkhalter et al., 1993; Callaway and Lieber, 1996; Callaway, 1998a). These studies have been interpreted to indicate that axonal arbors of layer $2 / 3$ pyramids develop with extreme precision from the outset, without making incorrect branches in layer 4 (Katz and Callaway, 1992). We show here, however, that in ferret visual cortex the very first axon collaterals that are formed by layer 2/3 pyramids (between $\mathrm{P} 14$ and P18) are not as specific as those that develop later. Furthermore, significant numbers of axonal branches that are formed in the middle of layer 4 are later eliminated. Careful comparison of these studies with previous studies in other species suggests that the early exuberance and later elimination of a small number of axonal branches in layer 4 could be a common feature of layer $2 / 3$ pyramids and may not be unique to ferrets (see further discussion below). These findings have important implications for understanding the mechanisms that give rise to the adult patterns and precision of layer-specific axonal arbors.

Studies of the axonal growth of layer $2 / 3$ pyramidal neurons in long-term (5 DIV) slice cultures revealed that the ability to grow specifically in the correct layers in vitro depends on the age of the animal when the slice cultures are made. When slice cultures are made after the time when layer-specific connections begin to form correctly in vivo (at P18 or later), in vitro growth of layer $2 / 3$ pyramidal cells is layer specific. If slice cultures are made from younger animals, in vitro growth lacks laminar specificity, just as it would in vivo. These observations indicate that the mechanisms that direct layer-specific axonal growth are preserved in cortical slice cultures and do not require extrinsic influences, such as patterned visual experience or connections with other structures. On the other hand, the selective elimination of axonal arbors in layer 4 that occurs in vivo did not occur in vitro, suggesting that the factors directing axon elimination from inappropriate layers are not retained in slices.

\section{Technical considerations}

Before discussing our results further, we will consider some technical aspects of our procedures. First, the "incorrect" axonal branches observed in layer 4 at P18 (24 hr cultures) are unlikely to have emerged during the $24 \mathrm{hr}$ in vitro, rather than previously in vivo. The specificity of axonal arbors from P18 slice cultures improves with further growth in vitro: after 5 DIV the laminar specificity of axonal branches is equal to or better than after $24 \mathrm{hr}$ (i.e., P18 versus P18 + 5 DIV) (Figs. 4A, 8B). Thus, if any changes were to occur within the first $24 \mathrm{hr}$, these would be likely to improve specificity rather than make it worse.

It is also possible that small errors in the assignment of laminar
A

\section{Apical Dendrite}

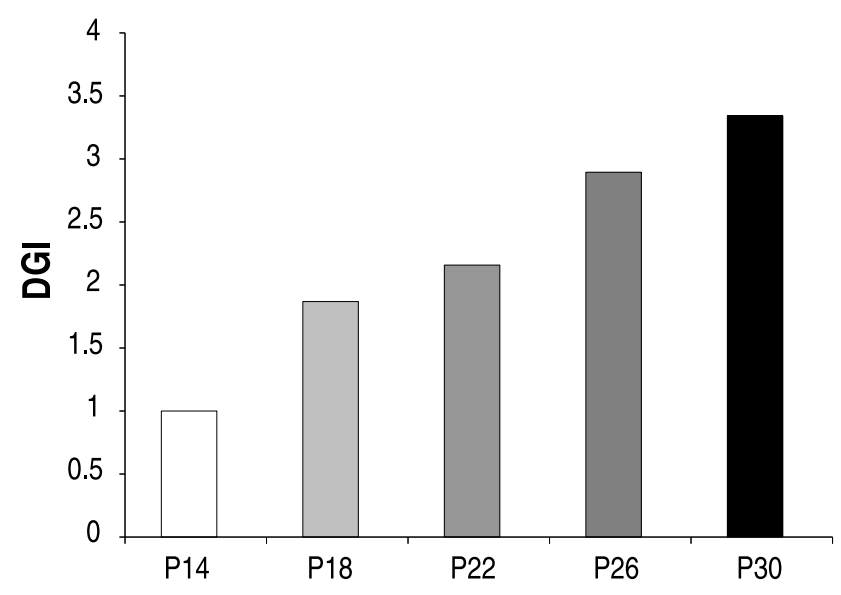

B

\section{Basal Dendrites}

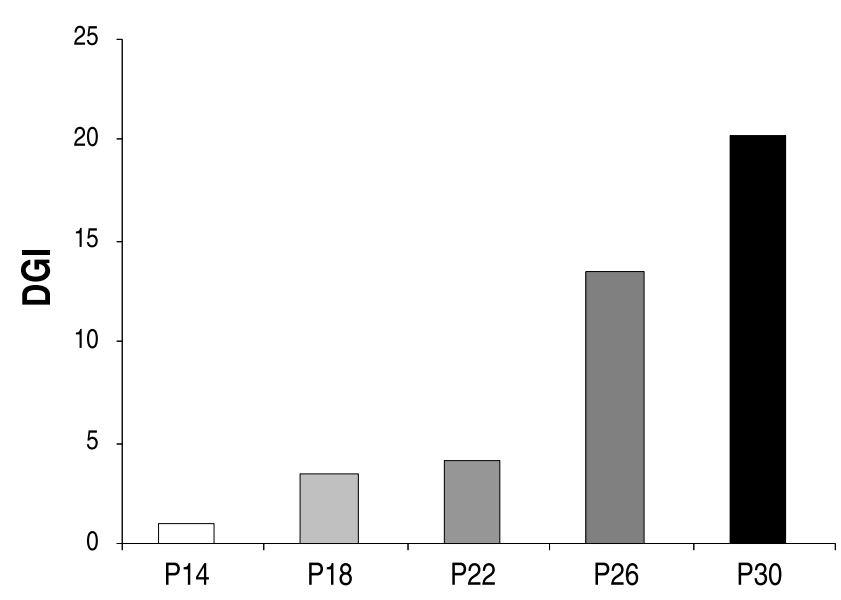

Figure 10. Histograms illustrating the changes in dendrite growth index (DGI; see Results) values of layer 2/3 pyramidal neurons between $P 14$ and P30. A DGI value of 1 means no significant change in dendritic arbor complexity (relative to complexity at P14). Larger values of DGI indicate greater increases in complexity. All ages were compared with P14; thus DGI $=1$ for this age. $A$, The DGI of apical dendrites showed a steady increase from $P 14$ to $P 30$. B, For basal dendrites, DGI values increased progressively between $P 14$ and $P 22$, but between $P 22$ and $P 26$ the DGI increased dramatically and increased still further until $P 30$. 
boundaries might have incorrectly assigned to layer 4 axonal branches that were actually located at the bottom of layer 3 or the top of layer 5. This cannot account, however, for all of the exuberant axonal branches that were detected in layer 4 at P18 or for the later elimination of these branches. We detected extensive decreases in axonal branches throughout the depth of layer 4, including the middle $60 \%$, and the most persistent decreases were found in the middle, not at the edges (Fig. $5 H$ ).

Axonal branches remained at the edges of layer 4 at the oldest age studied (P30), but these could be attributed almost entirely to neurons with cell bodies near the layer $3 / 4$ border (Fig. $4 D$ ), as observed previously in cat (Katz, 1991). This proximity to the border left the cells no choice but to branch in layer 4 or not at all. A similar constraint may contribute to the presence of branches at the edges of layer 4 in our P30 sample, but it is not the cause at P18 (Fig. 4, compare $B, D$ ). Thus, it is likely that from P18 to P30, neurons located farther from the layer $3 / 4$ border decreased their axonal branches not only in the middle of layer 4 but also at the edges.

\section{Transient formation and elimination of axonal branches in layer 4}

Previous studies have shown that in cats, monkeys, and humans, layer $2 / 3$ pyramidal neurons develop axonal arbors specifically in layers $2 / 3$ and 5 , whereas axonal branches were rarely or never observed in layer 4 (Katz, 1991; Burkhalter et al., 1993; Callaway, 1998a). No previous study has shown elimination of axonal branches of layer $2 / 3$ pyramids from layer 4 . Here we show that in the ferret visual cortex, layer $2 / 3$ pyramidal neurons form ectopic axonal branches in layer 4 transiently during development, and these are subsequently eliminated. Therefore the mechanisms that regulate axonal growth are not as precise initially as later, and mechanisms must exist to allow selective elimination of axon collaterals from incorrect layers.

Although we detect a decrease in the number of axon collaterals in layer 4 after P18, we cannot discern with certainty whether this is caused by retraction of axons or interstitial axon elongation, which could shift the position of existing branches out of layer 4. However, the shifting of axon positions is unlikely to be extensive enough to move axons out from the center of layer 4. Interstitial axon elongation is more likely to contribute to decreases near the edges of layer 4 , if it contributes at all. Thus, we favor the interpretation that axonal branches in the middle of layer 4 are retracted.

A likely explanation for the emergence of axonal branches in layer 4 between P14 and P18 is an absence of appropriate molecular signals. Numerous studies have suggested roles for extracellular ligands and their receptors in the precise development of axonal arbors (Bolz and Castellani, 1997; Mueller, 1999). The absence of branch-inhibiting extracellular ligands in layer 4 and their receptors in layer $2 / 3$ pyramidal cells might explain the erroneous formation of branches within layer 4 during this period. Similarly, the onset of expression of such molecules between P18 and P22 could prevent the later formation of additional branches.

To assess the possibility that exuberance and elimination of axonal branches in layer 4 is a general feature of the development of layer $2 / 3$ pyramids, rather than a feature unique to ferrets, it is instructive to compare our study with previous studies. The most detailed study was in the cat (Katz, 1991), which is closely related to the ferret but $\sim 2-3$ weeks more mature at birth (Luskin and Shatz, 1985; Jackson et al., 1989; Issa et al., 1999). The earliest age examined in the cat was P5, equivalent to approximately P22-P26 in ferrets, and at this time most layer $2 / 3$ pyramids already had axonal branches in layer $2 / 3$, as we also observe in P22 ferrets. However, our finding that branches in the middle of layer 4 are most prevalent at P18 (Fig. $5 H$ ) suggests that such branches might also be present in cats early in their first postnatal week (or during their last embryonic week). Katz (1991) suggested that the branches formed by layer $2 / 3$ pyramids were specific from the outset, even by the least mature neurons. We also observe no relationship between laminar specificity of axonal arbors and the birth date of neurons (inferred from their laminar position) (Fig. $4 E$ ), but we do observe an important influence of the age of the animal. These findings suggest that the maturation of features relevant to the development of layer-specific axons is regulated synchronously in all layer $2 / 3$ pyramids, independent of their birth date. Between P14 and P22 there is a developmental transition such that ectopic axonal branches are no longer formed in layer 4 but instead are eliminated.

\section{Coincidence of axonal and dendritic growth between P22 and P26}

In the present study we have shown that layer $2 / 3$ pyramidal neurons make relatively few axonal branches within layers $2 / 3$ and 5 between P14 and P22, but then exhibit a dramatic increase in branch formation in these layers during the subsequent $4 \mathrm{~d}$, which continues at least until P30. In addition, we have observed that development of the dendritic arbors of these neurons follows a similar time course. Apical and basal dendrites remain simple between P14 and P22, but between P22 and P26 they branch and elongate dramatically. Interestingly, layer 6 pyramids show a similar delay in axonal branching until P20, and then between P20 and P26 they display a burst of branch formation in layer 4 (Callaway and Lieber, 1996). Moreover, this timing is similar to that of layer 4 spiny stellate neurons in cat, which after a "latency" period between P5 and P11 (comparable to P22 to P28 in ferret), suddenly grow axons into layer 2/3 (Callaway and Katz, 1992). Taken together, these findings suggest the existence of an orchestrated regulation of axonal and dendritic growth starting at P22. Indeed, a "simultaneity rule" has previously been proposed as a mechanism for some aspects of cortical development (Katz and Callaway, 1992).

Very little is known about the molecules involved in the development of axonal laminar specificity in the cerebral cortex (Bolz and Castellani, 1997). The simultaneous growth of axonal and dendritic arbors shown in the present study suggests the existence of common signals regulating both processes (i.e., neurotrophins) (Cohen-Cory and Fraser, 1995; Inoue and Sanes, 1997; Martinez et al., 1998; Castellani and Bolz, 1999; Lom and Cohen-Cory, 1999; McAllister et al., 1999). It is also possible that the onset of visual activity at P23 in ferret (Chapman and Stryker, 1993) might be a factor influencing the expression of distinct molecular cues that independently regulate the development of axons and dendrites.

\section{REFERENCES}

Bolz J, Castellani V (1997) How do wiring molecules specify cortical connections? Cell Tissue Res 290:307-314.

Burkhalter A, Bernardo KL, Charles V (1993) Development of local circuits in human visual cortex. J Neurosci 13:1916-1931.

Butler AK, Dantzker JL, Shah RB, Callaway EM (2001) Development of visual cortical axons: layer-specific effects of extrinsic influences and activity blockade. J Comp Neurol 430:321-331.

Callaway EM (1998a) Prenatal development of layer-specific local circuits in primary visual cortex of the macaque monkey. J Neurosci 18:1505-1527. 
Callaway EM (1998b) Local circuits in primary visual cortex of the macaque monkey. Annu Rev Neurosci 21:47-74.

Callaway EM, Katz LC (1992) Development of axonal arbors of layer 4 spiny neurons in cat striate cortex. J Neurosci 12:570-582.

Callaway EM, Lieber JL (1996) Development of axonal arbors of layer 6 pyramidal neurons in ferret primary visual cortex. J Comp Neurol 376:295-305.

Callaway EM, Wiser AK (1996) Contributions of individual layer 2-5 spiny neurons to local circuits in macaque primary visual cortex. Vis Neurosci 13:907-922.

Castellani V, Bolz J (1999) Opposing roles for neurotrophin-3 in targeting and collateral formation of distinct sets of developing cortical neurons. Development 126:3335-3345.

Chapman B, Stryker MP (1993) Development of orientation selectivity in ferret visual cortex and effects of deprivation. J Neurosci 13:5251-5262.

Cohen-Cory S, Fraser SE (1995) Effects of brain-derived neurotrophic factor on optic axon branching and remodelling in vivo. Nature 378:192-196.

Dantzker JL, Callaway EM (1998) The development of local, layerspecific visual cortical axons in the absence of extrinsic influences and intrinsic activity. J Neurosci 18:4145-4154.

Gilbert CD (1983) Microcircuitry of the visual cortex. Annu Rev Neurosci $6: 217-247$.

Gilbert CD, Kelly JP (1975) The projections of cells in different layers of the cat's visual cortex. J Comp Neurol 163:81-105.

Goodman CS, Shatz CJ (1993) Developmental mechanisms that generate precise patterns of neuronal connectivity. Cell [Suppl] 72:77-98.

Inoue A, Sanes JR (1997) Lamina-specific connectivity in the brain: regulation by $\mathrm{N}$-cadherin, neurotrophins, and glycoconjugates. Science 276:1428-1431.

Issa NP, Trachtenberg JT, Chapman B, Zahs KR, Stryker MP (1999) The critical period for ocular dominance plasticity in the Ferret's visual cortex. J Neurosci 19:6965-6978.

Jackson CA, Peduzzi JD, Hickey TL (1989) Visual cortex development in the ferret. I. Genesis and migration of visual cortical neurons. J Neurosci 9:1242-1253.

Katz LC (1987) Local circuitry of identified neurons in cat visual cortex brain slices. J Neurosci 7:1223-1249.

Katz LC (1991) Specificity in the development of vertical connections in cat striate cortex. Eur J Neurosci 3:1-9.

Katz LC, Callaway EM (1992) Development of local circuits in mammalian visual cortex. Annu Rev Neurosci 15:31-56.

Lom B, Cohen-Cory S (1999) Brain-derived neurotrophic factor differentially regulates retinal ganglion cell dendritic and axonal arborization in vivo. J Neurosci 19:9928-9938.

Lund JS, Boothe R (1975) Interlaminar connections and pyramidal neuron organization in the visual cortex, area 17, of the macaque monkey. J Comp Neurol 159:304-334.

Lund JS, Boothe RG, Lund RD (1977) Development of neurons in the visual cortex (area 17) of the monkey (Macaca nemestrina): a Golgi study from fetal day 127 to postnatal maturity. J Comp Neurol 176:149-188.

Luskin MB, Shatz CJ (1985) Studies of the earliest generated cells of the cat's visual cortex: cogeneration of subplate and marginal zones. J Neurosci 5:1062-1075.

Martinez A, Alcantara S, Borrell V, Del Rio JA, Blasi J, Otal R, Campos N, Boronat A, Barbacid M, Silos-Santiago I, Soriano E (1998) TrkB and TrkC signaling are required for maturation and synaptogenesis of hippocampal connections. J Neurosci 18:7336-7350.

McAllister AK, Lo DC, Katz LC (1995) Neurotrophins regulate dendritic growth in developing visual cortex. Neuron 15:791-803.

McAllister AK, Katz LC, Lo DC (1999) Neurotrophins and synaptic plasticity. Annu Rev Neurosci 22:295-318.

Mueller BK (1999) Growth cone guidance: first steps towards a deeper understanding. Annu Rev Neurosci 22:351-388.

Tigges J, Tigges M (1982) Principles of axonal collateralization of laminae II-III pyramids in area 17 of squirrel monkey: a quantitative Golgi study. Neurosci Lett 29:99-104. .0 